\title{
Problem gambling in adolescents: what are the psychological, social and financial consequences?
}

\author{
Goran Livazović* (D) and Karlo Bojčić(D)
}

\begin{abstract}
Background: The paper examines the roles of sociodemographic traits, family quality and risk behaviour in adolescent problem gambling, with focus on the psychological, social and financial consequences from the socioecological model approach. This model emphasizes the most important risk-protective factors in the development and maintenance of problem gambling on an individual level, a relationship level, as well as a community and societal level.
\end{abstract}

Methods: The research was done using the Canadian Adolescent Gambling Inventory with a sample of 366 participants, 239 females (65.3\%) using descriptive statistics and t-test, ANOVA, correlation and hierarchical regression analysis.

Results: Males reported significantly higher gambling consequences on all scales $(p<.001)$ and significantly more risk behaviour $(p<.05)$. Age was significant for psychological consequences $(p<.01)$, problem gambling $(p<.01)$ and risk behaviour $(p<.001)$ with older participants scoring higher. Students with lower school success reported significantly higher psychological consequences of gambling $(p<.01)$, higher risk behaviour activity $(p<.001)$ and lower family life satisfaction $(p<.001)$. The psychological, financial and social consequences were positively correlated with problem gambling $(p<.001)$. Age $(p<.05)$, gender $(p<.001)$, school success $(p<.01)$ and the father's education level $(p .<05)$ were significant predictors of problem gambling, with older male adolescents who struggle academically and have lower educated fathers being at greater risk.

Conclusions: Results indicate an important relation between adolescent gambling behaviour and very serious psychological, social and financial consequences. There is a constellation of risk factors that likely place certain individuals at high risk for problem gambling.

Keywords: Gambling, Family, School, Adolescents, Risk behaviour, Sociodemographic traits

\section{Background}

In this article, we present a socio-ecological analysis of significant sociodemographic, family, school and gambling related factors predicting problem gambling among adolescents, as well as the most important empirical conclusions based on survey results with 366 participants, with special focus on the role of psychological, social and financial consequences, as well as riskprotective factors related to sociodemographic traits,

\footnotetext{
*Correspondence: glivazovic@ffos.hr; goran_livazovic@yahoo.com Department for Pedagogy, Faculty of Humanities and Social Sciences, University of J. J. Strossmayer in Osijek, Lorenz Jaeger Street 9, 31000 Osijek,
} Croatia

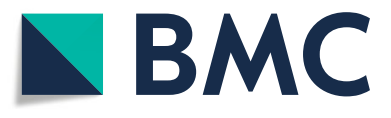

(c) The Author(s). 2019 Open Access This article is distributed under the terms of the Creative Commons Attribution 4.0 International License (http://creativecommons.org/licenses/by/4.0/), which permits unrestricted use, distribution, and reproduction in any medium, provided you give appropriate credit to the original author(s) and the source, provide a link to the Creative Commons license, and indicate if changes were made. The Creative Commons Public Domain Dedication waiver (http://creativecommons.org/publicdomain/zero/1.0/) applies to the data made available in this article, unless otherwise stated. family, school and adolescent risk behaviour. The socioecological model includes risk and protective factors on an individual level (health and personal traits), a relationship level (the closest social circle that contributes to the range of experience); the community level (the settings for interaction); and the societal level (social and cultural norms, as well as diverse social policies) [1]. The gambling panorama has shifted significantly during the past decades, from an initially mild type of entertainment to a hazardous addiction resulting in a number of academic, behavioural, personality, social, interpersonal, financial, criminal or mental health difficulties for children and adolescents experiencing gambling-related 
problems [2-4]. Current frameworks conceptualise problem gambling across a risk continuum [5], as the term describes gambling behaviour that results in adverse consequences for individuals, families and communities [6]. These consequences can range from impaired mental health, physical health, relationship and family dysfunction, to financial problems, employment difficulties and legal issues [7].

\section{Research on the characteristics and risk-protective factors in gambling}

Despite being illegal for minors, gambling is a common activity among adolescents. On an international level, estimates of past year gambling participation and problem gambling in youth (from 2000 to 2009) were highly variable, with rates of 0.8 to $6.0 \%$ [8], suggesting they exceed those of adults [9-12]. Researchers have reported evidence that a combination of biological, psychological and social factors contribute to gambling behaviour [13, 14]. A recent meta-analyses by Dowling et al. emphasized 13 individual risk factors (alcohol use frequency, antisocial behaviours, depression, male gender, cannabis use, illicit drug use, impulsivity, number of gambling activities, problem gambling severity, sensation seeking, tobacco use, violence, under-controlled temperament), one relationship risk factor (peer antisocial behaviours), one community risk factor (poor academic performance), one individual protective factor (socio-economic status) and two relationship protective factors (parent supervision, social problems) [12]. A number of problem gambling cross-sectional studies identified female gender, adaptive coping strategies, emotional intelligence, wellbeing, self-monitoring, personal competence, resilience, interpersonal skills, social competence, social support and bonding, school connectedness, parental monitoring and family cohesion as protective factors [3, 12, 15-22]. Integrated models of pathological gambling such as the Pathways model, introduced by Nower and Blaszczynski, suggest that a number of biological, personality, developmental, cognitive and environmental factors can be incorporated into a theoretical framework that helps explain youth gambling behaviour [23]. Derevensky et al. and Gupta and Derevensky suggest that in addition to helping youth understand the laws of probability, as well as independence of events and erroneous cognitions, attention must be paid to the underlying motivations leading to excessive adolescent pathological gambling (e.g., depressive symptomatology; somatic disorders; anxiety; attention deficits; self-regulation difficulties; academic, personal and familial problems; mood disorders; high risk-taking or poor coping skills) [24, 25]. Researchers also emphasize risk factors such as parental/familial gambling and parental/familial approval of gambling, antisocial behaviour, deviant peers, substance abuse and school problems [14, 26-30]. Problem gambling comorbid with mental health disorders has been associated with increased psychiatric symptoms; substance use problem severity; interpersonal, physical, financial and social difficulties; impulsivity and suicidality [31]. These factors play a role in the development and/or maintenance of gambling behaviour and problem gambling [27]. On the other hand, family life satisfaction (cohesion) and the quality of school affiliation, as indicators of social bonding, have been identified as protective factors in relation to youth gambling problems $[13,14,30]$.

\section{The present study}

This research was aimed on explicating the role of sociodemographic traits (age, gender, residence in urban or rural surroundings) in profiling and predicting the average problem gambler characteristics, as well as studying the impact of traditional protective factors such as family life quality (lower/higher personal satisfaction), family structure (both parents/ other), the parents' educational (lower/higher education) and professional status (employed/unemployed) and school related factors (school type, academic success). Special emphasis was placed on the relation between problem gambling behaviour and the psychological / social / financial consequences, as well as adolescent risk behaviour (physical violence/ alcohol/ drugs/ smoking/ risky sexual behaviour/school truancy/ deliberate destruction of property) from a predictive perspective. Based on the research aim and problems, the following hypotheses were established: (H1) sociodemographic traits have a significant impact on adolescent gambling; (H2) family life satisfaction, parental traits and school related factors represent protective factors in gambling aetiology; and (H3) problem gambling has significant psychological, social and financial consequences on adolescents.

\section{Methods \\ Study design and research goal}

The aim of the study was to examine the role of sociodemographic traits, family relations and parent characteristics, as well as school related factors in adolescent gambling behaviour with special focus on its psychological, social and financial consequences.

\section{Statistical analysis}

Quantitative analyses were conducted using descriptive and inferential procedures. The data was first processed for central tendency values on all measured items. The results were obtained using the t-test for independent samples and ANOVA concerning gender, age, place of residence, family structure, school success, school type, parents' educational level and the parents' employment status. A correlation analysis was implemented as to 
investigate the relation between sociodemographic traits, family life quality, risk behaviour, problem gambling and its psychological, social and financial consequences. A hierarchical regression analysis was implemented as to establish the most significant predictors for adolescent problem gambling. Due to a limited sample size and distribution, in order to perform the inferential tests, the "Age" variable was recoded into 2 groups, younger (14, 15 and 16) and older adolescents (ages 17-20). The variable "School success" was recoded into 3 groups, lower achievement (grades 1,2 and 3 or F, D, C), average achievement (grade 4 or B) and higher achievement (grade 5 or A). The variable "Parent employment" was recoded into 2 groups, the employed and unemployed/ other. The variable "Father's education level" and "Mother's education level" were both recoded into 2 groups, lower educated and higher educated fathers/ mothers. The data were tested using the Shapiro-Wilk normality test for age $(W=.821 ; p=, 000)$ and gender $(W=.602 ; p=, 000)$.

\section{Measures and data collection}

A multidimensional questionnaire was constructed for the purpose of this research. The five-degree pen-paper Likert-scale survey consisted of 4 parts in Croatian language.

a) The first part encompassed questions about sociodemographic traits (gender, age, school type, parent education level, family economic well-being, urban or rural residence, academic success, study programme).

b) The second part consisted of a non-standardised 4 item scale with questions on family life quality (i.e. $I$ get along with my parents; I can ask my parents for help; My family agrees on rules mutually), that was computed and transformed into a new composite variable named "Family life quality", with consequent reliability analysis showing a high Cronbach's alpha coefficient $(\alpha=.97)$.

c) The third part consisted of a non-standardised 8 item scale with questions on risk behaviours, which was computed and transformed into a new composite variable named "Risk behaviour" (i.e. I use physical violence to solve problems; I smoke cigarettes; I drink alcohol; I deliberately destroy property...), with consequent reliability analysis showing a satisfactory Cronbach's alpha coefficient $(\alpha=.70)$.

d) The last, fourth part of the questionnaire was based on the Canadian Adolescent Gambling Inventory [32, 33], with 3 standardised subscales on the financial consequences, the social consequences and the psychological consequences scale. The CAGI was developed specifically for adolescents [34, 35]. It is a paper-and-pen survey with 44-items, aimed at measuring the range and the complexity of gambling behaviour, rather than a dichotomy of either presence or absence of problem gambling, as is found in most existing adolescent and adult instruments [32, 33]. The CAGI has 19 items that measure gambling frequency using six-point response options and time spent gambling in a typical week on 19 forms of gambling and two items to measure money and items of value lost gambling [33]. It measures four gambling-related domains of loss of control, social, psychological and financial consequences, and a fifth, Gambling Problem Severity Scale (GPSS). In our study, the social consequences scale (i.e. I missed sports practice or other activity because of gambling; I missed a family gathering because of gambling...) had a satisfactory Cronbach's alpha coefficient $(\alpha=.67)$, the psychological consequences scale (i.e. I felt guilty for losing money on gambling; I felt sad or depressed because of the amount of money I lost gambling) had a high Cronbach's alpha coefficient $(\alpha=.93)$, and the financial consequences scale (i.e. I borrowed money for gambling; I stole to obtain money for gambling or repaying debts...) had a high Cronbach's alpha coefficient $(\alpha=.85)$, while the Gambling Problem Severity Scale (GPSS), (i.e. I planned gambling; After gambling, I returned to try win back the lost money...) also had a high Cronbach's alpha coefficient $(\alpha=.88)$. In previous research studies, CAGI was found to yield satisfactory estimates of reliability, validity and classification accuracy [32,33]. Studies have shown it is an appropriate instrument for epidemiological studies as well as for clinical and school settings [32-35].

\section{Participants}

A convenience sample was recruited to reflect the characteristics of the adolescent population. The research was conducted with 366 participants, 239 female and 127 male adolescents aged $14(N=35 ; 9.6 \%), 15(N=$ 142 ; 38.8\%), 16 ( $N=20$; $5.5 \%), 17(N=25 ; 6.8 \%), 18(N=$ 133; 36.3\%), $19(N=9 ; 2.5 \%)$ and $20(N=2 ; 0.5 \%)$. A total of $98(37.8 \%)$ participants reported living in rural areas, and 161 (62.2\%) lived in urban surroundings. 144 (39.3\%) participants attended gymnasiums, and 222 (60.7\%) vocational schools. 292 (79.8\%) reported living with both parents, 13 (3.6\%) only with their fathers, and $53(14.5 \%)$ with their mothers, while 8 (2.2\%) live with someone else. The 366 participants had a $4.1 \mathrm{GPA}$ with $32.2 \%(N=118)$ achieving an A (excellent), $53 \%(N=$ $194)$ achieving a $\mathrm{B}$ (very good), $12.6 \%(N=46)$ achieving a $C$ (good) and $2.2 \%(N=8)$ that failed class $(F)$. Participants reported that $6 \%(N=26)$ of their fathers completed only elementary school, 229 (62.6\%) finished high school, 47 (12.8\%) completed college and 63 (17.3\%) had university education. Some $6 \%(N=23)$ of mothers completed elementary school, 225 (61.5\%) finished high school, 42 (11.5\%) completed college and 76 (20.8\%) had university education. 


\section{Ethics and study procedure}

A paper-pen survey with high-school participants was conducted in March 2018 via group assessment during class in the Osječko-baranjska and Vukovarskosrijemska region in Croatia. Participants were introduced to the research goal prior to responding and given instructions on the procedure, as well as basic definitions on gambling behaviour. Individuals were excluded if they were unable to understand and provide informed consent. All participants were informed and guaranteed complete anonymity, in line with the Ethical Code of Research with Children [36]. Procedures performed in studies involving human participants were in accordance with the ethical standards of the institutional and/or national research committee, and with the 1964 Helsinki declaration and its later amendments or comparable ethical standards. A trained research assistant was responsible for the survey implementation. The research assistant was present at all times during the survey procedure and helped with possible explanations and survey guidelines. After completion, the participants were asked to check the survey answers as to guarantee responding to all the questions. The data was processed using SPSS (v20.0.0) with descriptive and inferential statistical analysis, t-test and one-way ANOVA, correlation analysis and hierarchical regression analysis.

\section{Results}

\section{Descriptive and inferential analysis}

\section{Sociodemographic characteristics and gambling}

The earliest reported age of gambling initiation was 7 $(N=1,0.3 \%)$, while gambling initiation was most prevalent at ages $14(N=13,3.6 \%), 15(N=10,2.7 \%)$ and 18 $(N=11,3 \%)$. Out of 366 participants, 154 (42.1\%) participants reported never having gambled. Our results show that playing cards, dares and challenges or skills represent the most prevalent adolescent gambling activities (Table 3$)$, while $6.6 \%(N=24)$ of them bet regularly in sports betting houses and $5.8 \%(N=21)$ do it online, $3 \%$ $(N=11)$ regularly bet on casino slot machines and $3.6 \%$ $(N=13)$ bet on live casino games, and 6.9\% $(N=25)$ bet on casino games online (Table 1).

Our t-test for gender differences results show that gen$d e r$ is a significant factor for psychological $(p<.001)$, social $(p<.001)$ and financial consequences $(p<.001)$ of gambling, as well as problem gambling $(p<.001)$ and risk behaviour $(p<.05)$, with males reporting significantly higher values on all scores (Table 2).

Our results were not significant for the social and financial consequences of gambling in relation to age, but showed significant age differences in psychological consequences $(p<.01)$, problem gambling behaviour $(p<.01)$ and risk behaviour $(p<.001)$ with older participants scoring higher (Table 3 ).
Our research has shown (Table 4) that lower school achievers report significantly higher psychological consequences of gambling in relation to more successful students $(p<.01)$.

Our results show no significant differences in gambling behaviour or its consequences in relation to the school type participants attend (Table 5), but indicate significantly higher risk behaviour for vocational school students $(p<.001)$.

Results on family and parent characteristics in gambling aetiology The place of residence (urban/ rural) was significant for the quality of family relations $\mathrm{t}(362)=10.03 ; p<.001$, with rural participants reporting significantly higher family life satisfaction $(N=98 ; \mathrm{M}=$ 12.07), but more risk behaviour as well, $\mathrm{t}(362)=2.26$, $p<.05$. Family structure (both parents/other) was significant for family life quality $\mathrm{t}(362)=-2.35, p<.05$, with participants from structurally deficient families reporting higher family life satisfaction $(N=74, M=8.74)$, and for risk behaviour $\mathrm{t}(362)=-2.50, p<.01$, as participants from structurally deficient families reported more risky behaviour $(N=74, \mathrm{M}=11.90)$. Our results show the mothers' employment status was significant only for family life satisfaction, as those who had employed mothers reported more satisfaction $\mathrm{t}(362)=3.71, p<.001$ $(N=245, \mathrm{M}=8.01)$. The fathers' employment status was not significant for any of the examined variables. Both the mothers and fathers' educational level did not show significance in relation to any of the examined variables.

\section{Correlation and regression analysis results for problem gambling}

Our results (Table 6) show a moderate to strong positive correlation between the psychological, financial and social consequences with problem gambling $(p<.001)$. There was a positive moderate correlation between problem gambling and risk behaviour, as well $(p<.001)$. Participants reported a positive weak correlation between school success and the psychological $(p<.001)$ and social consequences of problem gambling $(p<.01)$.

Our hierarchical regression analysis results show that age $(p<.05)$, gender $(p<.001)$, school success $(p<.01)$ and the father's education level $(p .<05)$ are significant sociodemographic predictors of problem gambling in adolescents, with older male adolescents who struggle academically and have lower educated fathers being at greater risk (Table 7). Interestingly, our results show that more psychological, social and financial consequences in gambling positively predict problem gambling $(p<.001)$.

\section{Discussion}

This study was focused on explicating the role of sociodemographic traits (age, gender, residence in urban or 
Table 1 Descriptive analysis on adolescent gambling preferences

\begin{tabular}{|c|c|c|c|c|c|c|c|c|}
\hline Variable & Group & & never & rarely & sometimes & often & always & $\Sigma$ \\
\hline \multirow[t]{4}{*}{ Playing cards } & Male & $\mathrm{N}$ & 62 & 17 & 20 & 12 & 16 & 127 \\
\hline & & $\%$ & 16.9 & 4.6 & 5.5 & 3.3 & 4.4 & 34.7 \\
\hline & Female & $\mathrm{N}$ & 179 & 28 & 18 & 8 & 6 & 239 \\
\hline & & $\%$ & 48.9 & 7.7 & 4.9 & 2.2 & 1.6 & 65.3 \\
\hline \multirow[t]{2}{*}{$\Sigma$} & & $\mathrm{N}$ & 241 & 45 & 38 & 20 & 22 & 366 \\
\hline & & $\%$ & 65.8 & 12.3 & 10.4 & 5.5 & 6 & 100 \\
\hline \multirow[t]{4}{*}{ Sports betting house } & Male & $\mathrm{N}$ & 79 & 9 & 18 & 10 & 11 & 127 \\
\hline & & $\%$ & 21.6 & 2.5 & 4.9 & 2.7 & 3 & 34.7 \\
\hline & Female & N & 225 & 8 & 3 & 3 & 0 & 239 \\
\hline & & $\%$ & 61.5 & 2.2 & 0.8 & 0.8 & 0 & 65.3 \\
\hline \multirow[t]{2}{*}{$\Sigma$} & & $\mathrm{N}$ & 304 & 17 & 21 & 13 & 11 & 366 \\
\hline & & $\%$ & 83.1 & 4.6 & 5.7 & 3.6 & 3.0 & 100 \\
\hline \multirow[t]{4}{*}{ Slot machines at casinos } & Male & N & 96 & 11 & 12 & 4 & 4 & 127 \\
\hline & & $\%$ & 26.2 & 3 & 3.3 & 1.1 & 1.1 & 34.7 \\
\hline & Female & $\mathrm{N}$ & 234 & 1 & 1 & 3 & 0 & 239 \\
\hline & & $\%$ & 63.9 & 0.3 & 0.3 & 0.8 & 0 & 65.3 \\
\hline \multirow[t]{2}{*}{$\Sigma$} & & N & 330 & 12 & 13 & 7 & 4 & 366 \\
\hline & & $\%$ & 90.2 & 3.3 & 3.6 & 1.9 & 1.1 & 100 \\
\hline \multirow[t]{4}{*}{ Live games at casinos } & Male & N & 99 & 9 & 8 & 6 & 5 & 127 \\
\hline & & $\%$ & 27 & 2.5 & 2.2 & 1.6 & 1.4 & 34.7 \\
\hline & Female & $\mathrm{N}$ & 232 & 3 & 2 & 2 & 0 & 239 \\
\hline & & $\%$ & 63.4 & 0.8 & 0.5 & 0.5 & 0 & 65.3 \\
\hline \multirow[t]{2}{*}{$\Sigma$} & & $\mathrm{N}$ & 331 & 12 & 10 & 8 & 5 & 366 \\
\hline & & $\%$ & 90.4 & 3.3 & 2.7 & 2.2 & 1.4 & 100 \\
\hline \multirow[t]{4}{*}{ Internet sports betting house } & Male & $\mathrm{N}$ & 93 & 9 & 7 & 6 & 12 & 127 \\
\hline & & $\%$ & 25.4 & 2.5 & 1.9 & 1.6 & 3.3 & 34.7 \\
\hline & Female & $\mathrm{N}$ & 229 & 6 & 1 & 3 & 0 & 239 \\
\hline & & $\%$ & 62.6 & 1.6 & 0.3 & 0.8 & 0 & 65.3 \\
\hline \multirow[t]{2}{*}{$\Sigma$} & & $\mathrm{N}$ & 322 & 15 & 8 & 9 & 12 & 366 \\
\hline & & $\%$ & 88 & 4.1 & 2.2 & 2.5 & 3.3 & 100 \\
\hline \multirow[t]{4}{*}{ Internet casino games } & Male & N & 84 & 11 & 14 & 10 & 8 & 127 \\
\hline & & $\%$ & 23 & 3 & 3.8 & 2.7 & 2.2 & 34.7 \\
\hline & Female & N & 206 & 17 & 9 & 6 & 1 & 239 \\
\hline & & $\%$ & 56.3 & 4.6 & 2.5 & 1.6 & 0.3 & 65.3 \\
\hline \multirow[t]{2}{*}{$\Sigma$} & & $\mathrm{N}$ & 290 & 28 & 23 & 16 & 9 & 366 \\
\hline & & $\%$ & 79.2 & 7.7 & 6.3 & 4.4 & 2.5 & 100 \\
\hline \multirow[t]{4}{*}{ Own or someone else's performance in games of skill } & Male & $\mathrm{N}$ & 47 & 29 & 24 & 19 & 8 & 127 \\
\hline & & $\%$ & 12.8 & 7.9 & 6.6 & 5.2 & 2.2 & 34.7 \\
\hline & Female & $\mathrm{N}$ & 141 & 50 & 23 & 17 & 8 & 239 \\
\hline & & $\%$ & 38.5 & 13.7 & 6.3 & 4.6 & 2.2 & 65.3 \\
\hline \multirow[t]{2}{*}{$\Sigma$} & & $\mathrm{N}$ & 188 & 79 & 47 & 36 & 16 & 366 \\
\hline & & $\%$ & 51.4 & 21.6 & 12.8 & 9.8 & 4.4 & 100 \\
\hline \multirow[t]{2}{*}{ A dare or challenge that you or someone else can do something } & Male & $\mathrm{N}$ & 58 & 22 & 27 & 12 & 8 & 127 \\
\hline & & $\%$ & 15.8 & 6 & 7.4 & 3.3 & 2.2 & 34.7 \\
\hline
\end{tabular}


Table 1 Descriptive analysis on adolescent gambling preferences (Continued)

\begin{tabular}{|c|c|c|c|c|c|c|c|c|}
\hline Variable & Group & & never & rarely & sometimes & often & always & $\Sigma$ \\
\hline & Female & $\mathrm{N}$ & 162 & 34 & 22 & 15 & 6 & 239 \\
\hline & & $\%$ & 44.3 & 9.3 & 6 & 4.1 & 1.6 & 65.3 \\
\hline \multirow[t]{2}{*}{$\Sigma$} & & N & 220 & 56 & 49 & 27 & 14 & 366 \\
\hline & & $\%$ & 60.1 & 15.3 & 13.4 & 7.4 & 3.8 & 100 \\
\hline \multirow[t]{4}{*}{ Video games for money or something of value } & Male & N & 87 & 15 & 11 & 9 & 5 & 127 \\
\hline & & $\%$ & 23.8 & 4.1 & 3.0 & 2.5 & 1.4 & 34.7 \\
\hline & Female & $N$ & 218 & 12 & 4 & 1 & 4 & 239 \\
\hline & & $\%$ & 59.6 & 3.3 & 1.1 & 0.3 & 1.1 & 65.3 \\
\hline \multirow[t]{2}{*}{$\Sigma$} & & $N$ & 305 & 27 & 15 & 10 & 9 & 366 \\
\hline & & $\%$ & 83.3 & 7.4 & 4.1 & 2.7 & 2.5 & 100 \\
\hline
\end{tabular}

rural surroundings) in profiling and predicting the average problem gambler characteristics, as well as studying the impact of traditional protective factors such as family life quality, family structure, the parents' educational and professional status and school related factors. Special emphasis was placed on the relation between problem gambling behaviour and the psychological / social / financial consequences, as well as adolescent risk behaviour.

\section{Adolescent gambling preferences}

Derevensky and Gilbeau indicate that typical forms of teen gambling include: card playing for money (poker), sports wagering, dice and board games with family and friends; betting with peers on games of personal skill (e.g., pool, bowling, basketball); arcade or video games for money; purchasing lottery tickets; wagering at horse and dog tracks; gambling in bingo halls and card rooms; playing slot machines and table games in casinos; gambling on video lottery/poker terminals; wagering on the Internet; and placing bets with a bookmaker, recently mostly via the Internet or smartphones [14]. Our results show that playing cards, dares and challenges or skills represent the most prevalent adolescent gambling activities (Table 3$)$, while $6.6 \%(N=24)$ of them bet regularly in sports betting houses and $5.8 \%(N=21)$ do it online, $3 \%(N=11)$ regularly bet on casino slot machines and $3.6 \%(N=13)$ bet on live casino games, and $6.9 \%$ $(N=25)$ bet on casino games online.

Williams [37] has reported that gambling tendencies differed from study to study and location to location, as his study has shown that games of skill were the most popular gambling activity (51\%), followed by card games (47\%), sports betting (27\%) and dice games (24\%) [37]. This is not similar to some studies reporting greater rates of lottery participation by adolescents [38-40]. On the other hand, our present results are consistent with other studies in Alberta which found that adolescents favoured card games, games of skills, and sports betting as compared to other gambling activities [37, 41, 42]. Even though $23 \%$ of the Alberta sample reported one or

Table 2 T-test for gender differences

\begin{tabular}{|c|c|c|c|c|c|}
\hline Variable & Gender & $\mathrm{N}$ & Mean & SD & $t$ \\
\hline \multirow[t]{2}{*}{ Psychological consequences of gambling } & M & 127 & 6.48 & 3.76 & $7.18^{* * * *}$ \\
\hline & $\mathrm{F}$ & 239 & 4.44 & 1.68 & \\
\hline \multirow[t]{2}{*}{ Social consequences of gambling } & M & 127 & 4.72 & 1.69 & $4.18^{* * *}$ \\
\hline & $\mathrm{F}$ & 239 & 4.16 & .89 & \\
\hline \multirow[t]{2}{*}{ Financial consequences of gambling } & M & 127 & 4.42 & 1.29 & $3.89^{* * *}$ \\
\hline & $\mathrm{F}$ & 239 & 4.06 & .45 & \\
\hline \multirow[t]{2}{*}{ Family relations quality } & M & 127 & 7.35 & 6.45 & .32 \\
\hline & $\mathrm{F}$ & 239 & 7.13 & 6.26 & \\
\hline \multirow[t]{2}{*}{ Problem gambling } & M & 127 & 10.64 & 4.19 & $7.03^{* * *}$ \\
\hline & $\mathrm{F}$ & 239 & 8.47 & 1.66 & \\
\hline \multirow[t]{2}{*}{ Risk behaviour } & M & 127 & 11.61 & 4.74 & $2.61 *$ \\
\hline & $\mathrm{F}$ & 239 & 10.44 & 3.70 & \\
\hline
\end{tabular}

Note: ${ }^{*} p<.05 ;{ }^{* *} p<.01 ;{ }^{* * *} p<.001$ 
Table 3 T-test for age differences

\begin{tabular}{|c|c|c|c|c|c|}
\hline Variable & Age & $\mathrm{N}$ & Mean & SD & $\mathrm{t}$ \\
\hline \multirow[t]{2}{*}{ Psychological consequences of gambling } & Younger & 197 & 4.79 & 2.25 & $-2.65^{* *}$ \\
\hline & Older & 169 & 5.56 & 3.23 & \\
\hline \multirow[t]{2}{*}{ Social consequences of gambling } & Younger & 197 & 4.27 & 1.15 & -1.33 \\
\hline & Older & 169 & 4.45 & 1.37 & \\
\hline \multirow[t]{2}{*}{ Financial consequences of gambling } & Younger & 197 & 4.13 & .83 & -1.24 \\
\hline & Older & 169 & 4.24 & .88 & \\
\hline \multirow[t]{2}{*}{ Family relations quality } & Younger & 197 & 7.55 & 6.18 & 1.12 \\
\hline & Older & 169 & 6.81 & 6.48 & \\
\hline \multirow[t]{2}{*}{ Problem gambling } & Younger & 197 & 8.79 & 2.05 & $-2.99 * *$ \\
\hline & Older & 169 & 9.72 & 3.74 & \\
\hline \multirow[t]{2}{*}{ Risk behaviour } & Younger & 197 & 9.93 & 3.98 & $-4.70^{* * *}$ \\
\hline & Older & 169 & 11.91 & 4.05 & \\
\hline
\end{tabular}

Note: ${ }^{*} p<.05 ;{ }^{* *} p<.01 ;{ }^{* * *} p<.001$

more symptoms of problem gambling, only $2.5 \%$ met actual criteria for problem gambling [37]. This prevalence rate is lower than found in most other studies [40, 43-48]. It is also lower than the Shaffer et al. meta-analysis which reported prevalence rates of adolescent problem gambling to be $3.9 \%[49]$.

\section{The role of sociodemographic traits in adolescent} problem gambling

Our first hypothesis (H1) assumed that sociodemographic traits have a significant impact on adolescent gambling. Our results show distinct gender differences (Table 2), with males reporting significantly higher gambling consequences on all scales $(p<.001)$, but risk behaviour, as well $(p<.05)$. Previous research established a relationship between male gender and gambling or problem gambling [42, 44, 46, 48, 50-53]. Sheela et al. found gender was significantly associated with adolescents' gambling behaviour, with males having nearly three times the odds of being gamblers compared to girls [54]. A study by Di Nicola et al. has shown higher prevalence in gambling frequency among males, and significant

Table 4 ANOVA for differences in school success

\begin{tabular}{|c|c|c|c|c|c|c|c|}
\hline Variable & School success & $\mathrm{N}$ & Mean & SD & Min & Max & $\mathrm{F}$ \\
\hline \multirow[t]{3}{*}{ Psychological consequences } & Lower & 54 & $6.24^{*}>2.3$ & 3.46 & 4.00 & 18.00 & $6.14^{* *}$ \\
\hline & Medium & 194 & 5.13 & 2.81 & 4.00 & 20.00 & \\
\hline & Higher & 118 & 4.67 & 2.16 & 4.00 & 19.00 & \\
\hline \multirow[t]{3}{*}{ Social consequences } & Lower & 54 & 4.74 & 1.79 & 4.00 & 13.00 & 3.02 \\
\hline & Medium & 194 & 4.28 & 1.04 & 4.00 & 12.00 & \\
\hline & Higher & 118 & 4.31 & 1.28 & 4.00 & 14.00 & \\
\hline \multirow[t]{3}{*}{ Financial consequences } & Lower & 54 & 4.26 & .73 & 4.00 & 7.00 & .71 \\
\hline & Medium & 194 & 4.21 & 1.00 & 4.00 & 12.00 & \\
\hline & Higher & 118 & 4.11 & .61 & 4.00 & 10.00 & \\
\hline \multirow[t]{3}{*}{ Family relations quality } & Lower & 54 & 5.19 & 6.48 & .00 & 15.00 & $9.95^{* * *}$ \\
\hline & Medium & 194 & $8.55^{*}>1.3$ & 6.04 & .00 & 15.00 & \\
\hline & Higher & 118 & 5.94 & 6.24 & .00 & 15.00 & \\
\hline \multirow[t]{3}{*}{ Problem gambling } & Lower & 54 & 9.78 & 3.59 & 8.00 & 30.00 & 1.37 \\
\hline & Medium & 194 & 9.23 & 3.03 & 8.00 & 25.00 & \\
\hline & Higher & 118 & 8.97 & 2.57 & 8.00 & 21.00 & \\
\hline \multirow[t]{3}{*}{ Risk behaviour } & Lower & 54 & 12.17 & 4.36 & 6.00 & 23.00 & $8.77^{* * *}$ \\
\hline & Medium & 194 & 11.19 & 3.91 & 6.00 & 23.00 & \\
\hline & Higher & 118 & $9.65^{*}<1.2$ & 4.10 & 6.00 & 31.00 & \\
\hline
\end{tabular}


Table 5 T-test for differences in school type

\begin{tabular}{|c|c|c|c|c|c|}
\hline Variable & School type & $\mathrm{N}$ & Mean & SD & $\mathrm{t}$ \\
\hline \multirow[t]{2}{*}{ Psychological consequences of gambling } & Gymnasium & 144 & 4.82 & 2.50 & \multirow[t]{2}{*}{-1.82} \\
\hline & Vocational school & 222 & 5.36 & 2.92 & \\
\hline \multirow[t]{2}{*}{ Social consequences of gambling } & Gymnasium & 144 & 4.35 & 1.45 & \multirow[t]{2}{*}{-.01} \\
\hline & Vocational school & 222 & 4.36 & 1.12 & \\
\hline \multirow[t]{2}{*}{ Financial consequences of gambling } & Gymnasium & 144 & 4.09 & .45 & \multirow[t]{2}{*}{-1.55} \\
\hline & Vocational school & 222 & 4.24 & 1.03 & \\
\hline \multirow[t]{2}{*}{ Family relations quality } & Gymnasium & 144 & 5.14 & 5.92 & \multirow[t]{2}{*}{$-5.22^{* * *}$} \\
\hline & Vocational school & 222 & 8.55 & 6.22 & \\
\hline \multirow[t]{2}{*}{ Problem gambling } & Gymnasium & 144 & 8.96 & 2.84 & \multirow[t]{2}{*}{-1.37} \\
\hline & Vocational school & 222 & 9.39 & 3.07 & \\
\hline \multirow[t]{2}{*}{ Risk behaviour } & Gymnasium & 144 & 10.25 & 4.37 & \multirow[t]{2}{*}{$-2.22^{*}$} \\
\hline & Vocational school & 222 & 11.23 & 3.92 & \\
\hline
\end{tabular}

Note: ${ }^{*} p<.05 ;{ }^{* *} p<.01 ;{ }^{* * *} p<.001$

gender differences in maladaptive gambling behaviour, with males scoring higher on the SOGS-RA score (South Oaks Gambling Screen: Revised for adolescents) [55]. Similarly, Elton-Marshall, Leatherdale and Turner have shown that online and land-based gambling was significantly more popular among males [56]. MuttiPacker et al. indicated that gender was a significant predictor of baseline levels of gambling, as females reported lower initial levels of gambling problems [57], while studies by Williams [37] and Gonzalez-Roz et al. [58]

Table 6 Correlation matrix analysis

\begin{tabular}{|c|c|c|c|c|c|c|c|c|c|c|c|c|}
\hline Variable & & 1 & 2 & 3 & 4 & 5 & 6 & 7 & 8 & 9 & 10 & 11 \\
\hline \multirow[t]{2}{*}{1 PSYCH CONSEQ GAMBLING } & $r$ & - & $.58^{* * *}$ & $.28^{*^{* *}}$ & $.16^{* *}$ & $.67^{* * *}$ & $.27^{* * *}$ & $.20^{* * *}$ & $-.35^{* * *}$ & -.01 & -.02 & $.21^{* * *}$ \\
\hline & $\mathrm{N}$ & 366 & 366 & 366 & 366 & 366 & 366 & 366 & 366 & 366 & 366 & 366 \\
\hline \multirow[t]{2}{*}{2 SOCIAL CONSEQ GAMBLING } & r & $.58^{* * *}$ & - & $.39^{* * *}$ & -.01 & $.72^{* * *}$ & $.22^{* * *}$ & $.13^{*}$ & $-.21^{* * *}$ & .02 & -.04 & $.14^{* *}$ \\
\hline & N & 366 & 366 & 366 & 366 & 366 & 366 & 366 & 366 & 366 & 366 & 366 \\
\hline \multirow[t]{2}{*}{3 FINANC CONSEQ GAMBL } & r & $.28^{* * *}$ & $.39^{* * *}$ & - & .028 & $.66^{* * *}$ & $.17^{* *}$ & .091 & $-.20^{* * *}$ & .05 & .02 & .06 \\
\hline & N & 366 & 366 & 366 & 366 & 366 & 366 & 366 & 366 & 366 & 366 & 366 \\
\hline \multirow[t]{2}{*}{4 FAMILY RELATION QUALITY } & r & $.6^{* *}$ & -.01 & .03 & - & .06 & $.49^{* * *}$ & -.01 & -.02 & -.02 & $-.17^{* *}$ & -.01 \\
\hline & N & 366 & 366 & 366 & 366 & 366 & 366 & 366 & 366 & 366 & 366 & 366 \\
\hline \multirow[t]{2}{*}{5 PROBLEM GAMBLING } & r & $.67^{* * *}$ & $.72^{* * *}$ & $.66^{* * *}$ & .06 & - & $.26^{* * *}$ & $.19^{* * *}$ & $-.35^{* * *}$ & .04 & .000 & .08 \\
\hline & N & 366 & 366 & 366 & 366 & 366 & 366 & 366 & 366 & 366 & 366 & 366 \\
\hline \multirow[t]{2}{*}{6 RISK BEHAVIOUR } & r & $.27^{* * *}$ & $.22^{* * *}$ & $.17^{* *}$ & $.49^{* * *}$ & $.26^{* * *}$ & - & $.16^{* *}$ & $-.12^{*}$ & .08 & $-.12^{*}$ & $.16^{* *}$ \\
\hline & N & 366 & 366 & 366 & 366 & 366 & 366 & 366 & 366 & 366 & 366 & 366 \\
\hline \multirow[t]{2}{*}{$7 \mathrm{AGE}$} & r & $.20^{* * *}$ & $.13^{*}$ & .09 & -.01 & $.19^{* * *}$ & $.16^{* *}$ & - & $-.14^{* *}$ & .06 & -.05 & $.15^{* *}$ \\
\hline & $\mathrm{N}$ & 366 & 366 & 366 & 366 & 366 & 366 & 366 & 366 & 366 & 366 & 366 \\
\hline \multirow[t]{2}{*}{8 GENDER } & r & $-.35^{* * *}$ & $-.21^{* * *}$ & $-.20^{* * *}$ & -.02 & $-.35^{* * *}$ & $-.12^{*}$ & $-.14^{* *}$ & - & .06 & .05 & $-.16^{* *}$ \\
\hline & $\mathrm{N}$ & 366 & 366 & 366 & 366 & 366 & 366 & 366 & 366 & 366 & 366 & 366 \\
\hline \multirow[t]{2}{*}{9 FATHER EMPLOYMENT } & r & -.01 & .02 & .05 & -.02 & .04 & .08 & .06 & .06 & - & $.13^{*}$ & $.11^{*}$ \\
\hline & N & 366 & 366 & 366 & 366 & 366 & 366 & 366 & 366 & 366 & 366 & 366 \\
\hline \multirow[t]{2}{*}{10 MOTHER EMPLOYMENT } & r & -.02 & -.04 & .02 & $-.17^{* *}$ & .01 & $-.12^{*}$ & -.05 & .05 & $.13^{*}$ & - & .01 \\
\hline & $\mathrm{N}$ & 366 & 366 & 366 & 366 & 366 & 366 & 366 & 366 & 366 & 366 & 366 \\
\hline \multirow[t]{2}{*}{11 SCHOOL SUCCESS } & r & $.20^{* * *}$ & $.14^{* *}$ & .06 & -.01 & .08 & $.16^{* *}$ & $.15^{* *}$ & $-.16^{* *}$ & $.11^{*}$ & .01 & - \\
\hline & $N$ & 366 & 366 & 366 & 366 & 366 & 366 & 366 & 366 & 366 & 366 & 366 \\
\hline
\end{tabular}

Note: $p<.05^{*} ; p<.01^{* *} ; p<.001^{* * *}$

Only significant coefficients equal to or higher than 0.5 were highlighted in bold. Also, significant coefficients with only negative significant prefixes for a certain variable (gender) were presented in bold, as it shows a trend in correlation 
Table 7 Regression analysis for Problem gambling $(N=366)$

\begin{tabular}{|c|c|c|c|c|c|c|}
\hline \multirow[t]{2}{*}{ Problem gambling } & \multicolumn{3}{|c|}{$\begin{array}{l}\text { Model } 1 \\
\text { (Soc.-demo. characteristics) }\end{array}$} & \multicolumn{3}{|c|}{$\begin{array}{l}\text { Model } 2 \\
\text { (Risk-protective factors) }\end{array}$} \\
\hline & $\bar{B}$ & SE(B) & $\beta$ & $\bar{B}$ & SE(B) & $\bar{\beta}$ \\
\hline$\overline{\text { Age }}$ & .64 & .27 & $.34^{*}$ & -.06 & .14 & -.03 \\
\hline Gender & -2.11 & .31 & $-.34^{* * *}$ & -.55 & .17 & $-.09 * *$ \\
\hline Father's education & -.36 & .17 & $-.11 *$ & -.12 & .09 & -.04 \\
\hline Mother's education & .15 & .17 & .05 & -.04 & .09 & -.01 \\
\hline Father's employment & .07 & .09 & .04 & .03 & .05 & .02 \\
\hline Mother's employment & .03 & .11 & .01 & .02 & .06 & .01 \\
\hline Grade / class & -1.19 & .86 & -.19 & .45 & .45 & .08 \\
\hline School success & -.08 & .19 & -.02 & -.34 & .10 & $-.09 * *$ \\
\hline Psychol. conseq. of gambling & & & & .34 & .04 & $.32^{* * *}$ \\
\hline Social conseq. of gambling & & & & .87 & .08 & $.37^{* * *}$ \\
\hline Financial conseq. of gambling & & & & 1.39 & .09 & $.39^{* * *}$ \\
\hline Family relations quality & & & & -0.02 & .01 & -.01 \\
\hline Risk behaviour & & & & .04 & .02 & .05 \\
\hline$R^{2}$ & .160 & & & .781 & & \\
\hline R Square Change & .160 & & & .621 & & \\
\hline Adjusted $\boldsymbol{R}^{2}$ & .141 & & & .773 & & \\
\hline$d f$ & 8,357 & & & 13,35 & & \\
\hline$F$ & $8.482^{* * *}$ & & & 96.55 & & \\
\hline$F$ for change in $R^{2}$ & $8.48^{* * *}$ & & & 199.7 & & \\
\hline
\end{tabular}

Note: $p<.05^{*} ; p<.01^{* *} ; p<.001^{* * *}$

showed no statistically significant differences between gender and gambling behaviour. However, a recent meta-analysis reported male gender to be among the strongest risk factors for problem gambling [12], which was confirmed in our present study results.

In their investigation on the role of age, Sheela et al. did not find significant associations between age and adolescent gambling behaviour [54], but an earlier age of first gambling activity was established as a risk factor [59]. Several research studies found no significant age differences in psychological consequences of gambling, social consequences of gambling, financial consequences of gambling and problem gambling behaviour, as well $[30,55]$, while McBride and Derevensky showed that a greater proportion of non-gamblers were under the age of 18 years, whereas a significantly greater proportion of social gamblers were aged 21-24 [60]. In a study conducted by Kristiansen and Jensen, the proportions of atrisk gamblers and problem gamblers were significantly higher among older age groups [61]. Kristiansen and Jensen reported a weak, non-significant relationship between age and the SOGS-RA score, with older respondents reporting more gambling problems [61]. This is consistent with our findings and could imply that gambling behaviour is related to personal maturation, more agency and social emancipation, but also indicate that preventive actions need to be targeted at specific age groups. As evidence indicates, wagering something of value on an uncertain event often begins as early as grade school [44], with age 11 being the average age of onset found in a couple of major studies [25, 47]. Still, our results show a worrying age decline as the earliest reported age of gambling initiation was 7 , while it was most prevalent at ages 14, 15 and 18, implying a need for an earlier onset and optimization of efficient prevention programs in schools and community activities.

\section{Family life, parental traits and school related factors in problem gambling}

The second hypothesis (H2) assumed that family life satisfaction, parental traits and school related factors represent protective factors in gambling aetiology. Our research has shown (Table 4) that lower school achievers report significantly higher psychological consequences of gambling in relation to better students $(p<.01)$. A large amount of international research has found that problem gamblers tend to be concentrated among those lacking college education, and who have dropped out of high school, while several studies have demonstrated correlations between higher spending on gambling and lower levels of education [62]. Gambling during adolescence has been linked with psychiatric, social, and substance 
misuse problems in adulthood [63]. Both recreational and problem gambling have been associated with adverse social functioning and mental health in adolescence including poor school performance and difficulties with aggression and mood [53, 64, 65]. Foster et al. also reported that students who gambled on school grounds had poorer academic performance [66], while a study by Gonzalez-Roz et al. showed no statistically significant differences between school success and gambling behaviour [58]. Our results also demonstrate significantly higher risk behaviour activity $(p<.001)$ and significantly lower family life satisfaction among participants who report the lowest school achievement $(p<.001)$, so the intensity of psychological consequences could also stem and be related to a feeling of rejection due to problems in family, school failure and risk behaviour participation.

Our results showed no significant differences in gambling behaviour or its consequences in relation to the school type participants attended, but indicated significantly higher risk behaviour for vocational school students. These results are in line with similar studies which have shown that vocational school students have significantly higher risk behaviour prevalence than gymnasium students, with no significant school type differences in gambling behaviour $[53,67]$.

The place of residence (urban/rural) was significant for the quality of family relations, with rural participants reporting both significantly higher family life satisfaction, but more risk behaviour as well. Family structure (both parents/other) was significant for both family life quality and risk behaviour, with participants from structurally deficient families reporting higher family life satisfaction and riskier behaviour. Similarly, Foster et al. also did not establish significant gambling differences in relation to family structure [66], but Canale et al. reported that two family characteristics increased adolescent gambling - living with unrelated others or a single parent, and in poor families [68]. Our results show the mothers' employment status was significant only for family life satisfaction, with participants who had employed mothers reporting more satisfaction. The fathers' employment status was not significant for any of the examined variables. Both the mothers and fathers' educational level did not show significance in relation to any of the examined variables. Even though our study did not investigate parental gambling behaviours, previous research has positively correlated parental gambling and adolescent gambling, with children of problem gamblers tending to gamble earlier than their peers $[10,44,52,69,70]$, implying the need to educate parents on family risk factors for problem gambling.

\section{The consequences of adolescent problem gambling}

The third hypothesis (H3) assumed gambling had significant psychological, social and financial consequences on adolescents. Our results established a positive correlation between the psychological, financial and social consequences with problem gambling (Table 6), consistent with previous research on emotional problems and gambling, for example a positive relation between time spent gambling and depression [52, 71], also found in a study by Williams [37] and Rossen et al. [72], who reported that students with unhealthy gambling practices reported significantly more mental health issues and other addictions/risky behaviours [72]. Among the highrisk behaviours, adolescents that smoke, consume alcohol and participate in physical fights had significantly higher odds for gambling addiction. In a study by Castrén et al. both smoking and drinking for intoxication were significantly associated with at-risk and problem gambling compared with non-smokers and respondents who had not been drinking for intoxication [73]. Gambling frequency has been found to be highly associated with other forms of antisocial activity, for example, delinquency [74]. Additionally, Williams [37] points to a number of studies that reported a positive relationship between risk-taking and gambling, which was confirmed by a meta-analysis by Dowling et al. [12].

\section{Predictors of adolescent problem gambling}

Our hierarchical regression analysis results show that age, gender, school success and the father's education level all significantly predicted problem gambling in adolescents, with older male adolescents who struggle academically and have lower educated fathers being at greater risk (Table 7). Our results also established that more intense psychological, social and financial consequences in gambling positively predicted problem gambling. For example, Gupta and Derevensky [9] reported that excessive gambling in boys caused emotion-focused coping strategies, such as anger, frustration or anxiety during negative events. Similarly, Williams found a positive relationship between time spent gambling and depression [37]. All our regression results are in line with Rossen et al., who found that males are disproportionately at risk of problem gambling [72]. The same study found that students with unhealthy gambling practices were significantly more likely to report co-existing mental health issues (e.g. depression and suicide attempts) and other addictions/risky behaviours (e.g. use of alcohol and weekly cigarette smoking). For example, Williams found that time spent gambling was a significant predictor for higher levels of impulsivity, possessing more positive attitudes towards gambling, having been in trouble with the police, having suffered from depression, possessing less knowledge about gambling and having greater cognitive errors [37]. While Ste-Marie, Gupta and Derevensky found that gamblers with the highest scores on state and trait anxiety, as well as for social 
stress, were likely to meet the criteria for probable pathological gambling [28], a recent meta-analysis on problem gambling revealed that aggression, anxiety symptoms, attention problems, a big early gambling win, dispositional attention, psychological distress (including internalising symptoms), religious attendance and suicidal ideation were not significantly associated with subsequent problem gambling, so results are inconclusive [12]. Even though our results did not show a significant role of family structure in gambling, a study conducted by Allami et al. has shown that, at age of 16, parentchild connectedness and higher parental involvement significantly predicted fewer gambling problems, while peer connectedness may have an effect on problem gambling, but that effect likely depends on peer gambling behaviour [75]. A recent meta-analysis on gambling established parent supervision and socio-economic status as significantly negatively associated with subsequent problem gambling [12]. Our regression analysis did not establish family life quality or parent employment as significant predictors, while Jackson et al. found that participating in gambling activities was associated with parental employment [76]. They found no significant associations between gambling and family structure, nor between gambling and parental education. The same study also found a positive association between gambling involvement and depressive symptomology, deliberate self-harm and arguments with others; as it did between gambling participation and engagement in substance use and antisocial behaviours. These behaviours, with the exception of smoking, were significant predictors of greater involvement in gambling [76]. Interestingly, a study by Williams established a positive attitude toward gambling as the most consistent predictor of gambling behaviour, as well as problem gambling [37]. Among other established predictors, Williams found that larger amounts of money won while gambling, positive attitudes towards gambling, impulsivity, more gamblingrelated cognitive errors, greater risk-taking and less knowledge about gambling, were the variables that significantly contributed to the prediction of higher gambling frequency in order of predictability [37]. Similarly, having won a large sum of money gambling was the best predictor of increased time spent gambling. In addition, spending more time gambling was also associated with higher levels of impulsivity, possessing more positive attitudes towards gambling, having been in trouble with the police, having suffered from depression, possessing less knowledge about gambling and having greater cognitive errors, in descending order of predictability [37]. Reductions in positive attitudes towards gambling, prior trouble with the police and increased knowledge were the only three variables that significantly predicted decreased gambling frequency [37]. Surprisingly, even though we did not establish predictive relations between risky behaviour and problem gambling, it has been found to be highly associated with other forms of antisocial activity. For example, delinquency has been found to be positively related to both gambling frequency and problem gambling [73].

\section{Implications for research and practice}

Problem gambling is a complex research area, so findings are sometimes contradictory even though such behaviour is proven to disrupt personal, family, financial, professional and social relations [62]. Our study was focused on explicating the role of sociodemographic traits, family relations quality and risk behaviour in adolescent problem gambling. Such quantitative studies on sociodemographic factors related to personal characteristics, family life satisfaction and school surroundings are relatively scarce. While many adolescents gamble occasionally and don't experience significant problems, studies suggest they constitute a vulnerable population for gambling problems, especially with the onset of online gambling. Low numbers of treatment seeking adolescent problem gamblers may be related to the fact that they do not consider disruptive gambling as problematic and underestimate its impact. Treatment-wise, high comorbidity with other disorders may obscure problem gambling, as other problems get more clinical attention, while the heterogeneity within a relatively narrow age range implies the need for divesified approaches [77]. Our results indicate $3-7 \%$ of adolescents who regularly participate in serious gambling activities, in line with a study by Volberg et al., who reported $2-8 \%$ of adolescents having serious gambling problems, with another $10-15 \%$ being at-risk for the development of a gambling problem, especially young adults ages 18-25 [78]. Our regression analysis showed that the average at-risk individuals are older male adolescents who struggle academically, have lower educated fathers, attend vocational schools and report low family life satisfaction. It is interesting we established that problem gambling was positively predicted by psychological, social and financial consequences, in accordance with studies that established high comorbidity of problem gambling with psychological conditions [79], but their relation remains unclear as to the nature or relationship of the three variables in the prevention or inhibition of gambling. Adolescent problem gamblers use less task-focused coping and more avoidance coping strategies than nongamblers, while male excessive gamblers demonstrate more emotion-focused coping strategies, such as anger, frustration or anxiety during negative events [80]. Therefore, gambling behaviour is closely related to feelings of serious social, psychological and financial consequences for adolescents. Similar to adults with a gambling 
disorder, adolescents report having a preoccupation with gambling; repeated attempts at recouping losses; increasing wagers to reach a physiological level of excitement; lying to others about gambling; with anxiety and depression when trying to reduce their gambling. A considerable number of adolescents report gambling as a coping mechanism to psychologically escape daily problems (parental, peer, and school-related) and other mental health issues [13, 81]. Surprisingly, family life quality, parent employment and risky behaviour were not established as significant predictors for problem gambling in our study, so the role of parents and family should be further investigated. Lussier et al. examined the concept of resilience for youth gambling problems and other adolescent high-risk behaviours and suggested that family cohesion was an important element in adolescent resilience [17]. Exposure to an object of addiction at a young age or exposure to a parent's addiction could both increase the likelihood of developing an addiction. One recent study found that children of pathological gamblers were four times more likely to develop the disorder, so it is important that health professionals, teachers and other experts understand the family background [82]. Recent findings suggest that some of the early factors associated with the onset of problem gambling in cross-sectional studies have not been identified in subsequent longitudinal studies, which suggests that these factors may be, in fact, consequences of problem gambling or co-exist because they share common causes [12]. Therefore, longitudinal studies shift the policy focus from elements that co-occur with problem gambling in youth at a certain cross-sectional point in time to predictive factors of gambling at a future time-point, including adulthood. Adolescent gambling behaviour should be viewed on a continuum, from non-gambling to social or occasional and recreational gambling to atrisk gambling, up to problem or pathological, compulsive or disordered gambling [14]. In conclusion, clinical and research evidence suggests that efficient prevention strategies have an impact through providing facts about gambling which improve knowledge and significantly reduce misconceptions, resulting in more realistic attitudes towards gambling $[83,84]$. Still, their effectiveness needs further investigation, thus emphasising the need for future efficient preventive social and educational policy.

\section{Limitations}

Several limitations of the current study should be noted. The research sample was small; therefore, conclusions of a larger scale and results generalization are out of the scope of this study. In addition, the extent of underreporting or over-reporting of behaviours cannot be determined, although the survey questions demonstrate good intercorrelational reliability. The "Gambling social consequences" scale demonstrated lower reliability, even though standardised survey instruments were implemented. Sociodemographic traits, family relations, risk behaviour and problem gambling experiences were self-reported, but previous studies have shown these measures to be valid [85, 86]. Despite these limitations, a strength of the study was the use of instruments with reliable psychometric properties to measure adolescent gambling behaviour and its social, psychological and financial consequences.

\section{Conclusions}

The results of our study indicate that there is a distinct role of socio-demographic characteristics in the aetiology of adolescent problem gambling, that are mostly related to gender, age, academic achievement and the father's educational level. What begins as an exciting benign form of entertainment for most, could result in serious problems for an identifiable group of young people. It should be noticed that our findings clearly indicate an important relation between adolescent gambling behaviour and very serious psychological, social and financial consequences, as well as risk behaviour. There is a constellation of factors that likely place certain individuals at high risk for problem gambling. The increasing awareness that the aetiology underlying gambling problems is not universal, that risk factors may be different for individuals, and that there are a number of distinct pathways which could lead to pathological or disordered gambling, pose new important questions for researchers and practitioners on the structure and focus of preventive activities.

\section{Acknowledgements}

Not applicable.

\section{Authors' contributions}

The first author, GL, envisioned the research framework and was involved in writing the theoretical part of the paper, conducted the empirical data analysis and discussion on the results of the study. The second author, KB, was involved with the practical research data gathering and survey implementation, organised the field research and was involved with the discussion on the results of the study. Both authors have read and approved the final manuscript.

\section{Funding}

No funding was obtained for this study.

\section{Availability of data and materials}

All research data is available upon demand, and was submitted to the Editorial Office in the publication process. Requests for data and materials should be addressed to the corresponding author.

\section{Ethics approval and consent to participate}

All procedures performed in studies involving human participants were in accordance with the ethical standards of the institutional and/or national research committee and with the 1964 Helsinki declaration and its later amendments or comparable ethical standards. The research was conducted according to The Ethical Standards for Research with Children (2003) and the standards of the Ethical Committee for Research of the Faculty of Humanities and Social Sciences in Osijek. Informed written consent was obtained from all individual participants included in the study, as well as their parents and school institutions. All supporting data can be accessed via e-mail, available on demand. 


\section{Consent for publication}

Not applicable.

\section{Competing interests}

The authors declare that they have no competing interests.

Received: 16 April 2019 Accepted: 16 September 2019 Published online: 22 October 2019

\section{References}

1. Dahlberg LL, Krug EG. Violence: a global public health problem. In: Krug EG, Dahlberg LL, Mercy JA, Zwi AB, Lozano R, editors. World report on violence and health. Geneva: World Health Organization; 2002. p. 1-56.

2. Hardoon K, Gupta R, Derevensky JL. Psychosocial variables associated with adolescent gambling: a model for problem gambling. Psychol Addict Behav. 2004; https://doi.org/10.1037/0893-164X.18.2.170.

3. Shead N, Derevensky JL, Gupta R. Risk and protective factors associated with youth problem gambling. Int J Adolesc Med Health. 2010;22(1):39-58.

4. Derevensky J. Teen gambling: understanding a growing epidemic. New York: Rowman \& Littlefield Publishing; 2012.

5. Shaffer HJ, Korn DA. Gambling and related mental disorders: A public health analysis. Annu Rev Public Health. 2002; https://doi.org/10.1146/annurev. publhealth.23.100901.140532.

6. Neal PN, Delfabbro PH, O'Neil MG. Problem gambling and harm: towards a national definition. Office of Gaming and Racing, Victorian Government Department of Justice: Melbourne; 2005

7. Shaffer HJ, Hall MN. The natural history of gambling and drinking problems among casino employees. J Soc Psychol. 2002; https://doi.org/10.1080/ 00224540209603909

8. Volberg RA, Gupta R, Griffiths MD, Olason DT, Delfabbro P. An international perspective on youth gambling prevalence studies. Int J Adolesc Med Health. 2011; https://doi.org/10.1515/IJAMH.2010.22.1.3.

9. Gupta R, Derevensky JL, Marget N. Coping strategies employed by adolescents with gambling problems. Child Adolesc Mental Health. 2004; https://doi.org/10.1111/j.1475-3588.2004.00092.x

10. Jacobs DF. Youth gambling in North America. In: Derevensky J, Gupta R, editors. Gambling problems in youth: theoretical and applied perspectives New York: Springer US; 2005. p. 1-24.

11. Huang $\mathrm{JH}$, Boyer R. Epidemiology of youth gambling problems in Canada: A national prevalence study. Can J Psychiatry. 2007; https://doi.org/10.1177\%2 F070674370705201006

12. Dowling NA, Merkouris SS, Greenwood CJ, Oldenhof E, Toumbourou JW, Youssef GJ. Early risk and protective factors for problem gambling: a systematic review and meta-analysis of longitudinal studies. Clin Psychol Rev. 2017; https://doi.org/10.1016/j.cpr.2016.10.008.

13. Sharpe $L$. A reformulated cognitive-behavioral model of problem gambling: a biopsychosocial perspective. Clin Psy Rev. 2002; https://doi.org/10.1016/ S0272-7358(00)00087-8.

14. Derevensky JL, Gilbeau L. Adolescent gambling: twenty-five years of research. Can J Addict. 2015;6(2):4-12.

15. Chalmers $\mathrm{H}$, Willoughby $\mathrm{T}$. Do predictors of gambling involvement differ across male and female adolescents? J Gambl Stud. 2006; https://doi.org/10. 1007/s10899-006-9024-6.

16. Dickson LM, Derevensky JL, Gupta R. The prevention of gambling problems in youth: a conceptual framework. J Gambl Stud. 2002; https://doi.org/10. 1023/A:1015557115049.

17. Lussier I, Derevensky JL, Gupta R, Bergevin T, Ellenbogen S. Youth gambling behaviors: An examination of the role of resilience. Psychol Addict Behav. 2007; https://doi.org/10.1037/0893-164X.21.2.165.

18. Magoon ME, Ingersoll GM. Parental modeling, attachment, and supervision as moderators of adolescent gambling. J Gambl Stud. 2006; https://doi.org/ 10.1007/s10899-005-9000-6.

19. Nower L, Derevensky J, Gupta R. The relationship of impulsivity, sensation seeking, coping, and substance use in youth gamblers. Psychol Addict Behav. 2004; https://doi.org/10.1037/0893-164X.18.1.49.

20. Parker JDA, Taylor RN, Eastabrook JM, Schell SL, Wood LM. Problem gambling in adolescence: Relationships with internet misuse, gaming abuse and emotional intelligence. Pers Individ Dif. 2008. https://doi.org/10.1016/j. paid.2008.03.018
21. Turner NE, Macdonald J, Bartoshuk M, Zangeneh M. Adolescent gambling behaviour, attitudes, and gambling problems. Int J Ment Health Addiction. 2008; https://doi.org/10.1007/s11469-007-9117-1.

22. Weinstock J, Petry NM. Pathological gambling college Students' perceived social support. J Coll Stud Dev. 2008;49(6):625-32.

23. Nower $L$, Blaszczynski A. A pathways approach to treating youth gamblers. In: Derevensky J, Gupta R, editors. Gambling problems in youth: theoretical and applied perspectives. New York: Springer US; 2005. p. 189-209.

24. Derevensky J, Temcheff C, Gupta R. Treatment of adolescent gambling problems: more art than science? In: Derevensky J, Shek DTL, Merrick J, editors. Youth gambling: the hidden addiction. Boston: De Gruyter; 2011. p. 167-86.

25. Gupta R, Derevensky JL. Adolescents with gambling problems: from research to treatment. J Gambl Stud. 2000; https://doi.org/10.1023/A: 1009493200768

26. Winters KC, Stinchfield RD, Botzet A, Anderson N. A Prospective Study of Youth Gambling Behaviors. Psychol Addict Behav. 2002; https://doi.org/10. 1037/0893-164X.16.1.3.

27. Stinchfield R. Demographic, psychosocial, and behavioral factors associated with youth gambling and problem gambling. In: Derevensky J, Gupta R, editors. Gambling problems in youth: theoretical and applied perspectives. New York: Springer US; 2005. p. 27-39.

28. Ste-Marie C, Gupta R, Derevensky JL. Anxiety and social stress related to adolescent gambling behavior and substance use. J Child Adolesc Subst Abuse. 2006; https://doi.org/10.1300/J029v15n04_03.

29. Zangeneh M, Grunfeld A, Koenig S. Individual factors in the development and maintenance of problem gambling. In: Zangeneh M, Blaszczynski A, Turner NE, editors. In the pursuit of winning: problem gambling theory, research and treatment. New York: Springer; 2008. p. 83-94.

30. Lussier ID, Derevensky JL, Gupta R, Vitaro F. Risk, Compensatory, Protective, and Vulnerability Factors Related to Youth Gambling Problems. Psychol Addict Behav. 2014; https://doi.org/10.1037/a0034259.

31. Manning V, Dowling NA, Lee S, Rodda S, Garfield JBB, Volberg R, Kulkarni J, Lubman DI. Problem gambling and substance use in patients attending community mental health services. J Behav Addict. 2017; https://doi.org/10. 1556/2006.6.2017.077.

32. Tremblay J, Stinchfield R, Wiebe J, Wynne H. Canadian Adolescent Gambling Inventory (CAGI): Phase III Final Report. Canadian Centre on Substance Abuse. 2010; http://www.ccgr.ca/en/projects/resources/CAGI-Phase-IIIReport-English.pdf. Accessed 7 Jun 2019.

33. Stinchfield R, Wynne H, Wiebe J, Tremblay J. Development and Psychometric Evaluation of the Brief Adolescent Gambling Screen (BAGS). Front Psychol. 2017; https://doi.org/10.3389/fpsyg.2017.02204.

34. Wiebe J, Wynne H, Stinchfield R, Tremblay J. Measuring problem gambling in adolescent populations: phase I report. Canadian Centre on Substance Abuse 2005. http://www.ccgr.ca/en/projects/resources/CAGI-Phase-l-Report. pdf. Accessed 7 Jun 2019.

35. Wiebe J, Wynne H, Stinchfield R, Tremblay J. The Canadian Adolescent Gambling Inventory (CAGI): Phase II Final Report. Canadian Centre on Substance Abuse. 2007. http://www.ccgr.ca/en/projects/resources/CAGlPhase-II-Report-English.pdf. Accessed 7 Jun 2019.

36. Ajduković M, Kolesarić V. Etički kodeks istraživanja s djecom. Zagreb: Vijeće za djecu Vlade Republike Hrvatske, Državni zavod za zaštitu obitelji, materinstva i mladeži; 2003.

37. Williams R. Prevention of problem gambling: a school-based intervention. Alberta Gaming Research Institute 2002. https://opus.uleth.ca/bitstream/ handle/10133/370/Williams_prevention.pdf. Accessed 10 Apr 2019.

38. Ide-Smith SG, Lea SEG. Gambling in young adolescents. J Gambl Stud. 1988; https://doi.org/10.1007/BF01020107.

39. Ladouceur R, Mireault C. Gambling behaviors among high school students in the Quebec area. J Gambl Stud. 1988; https://doi.org/10. 1007/BF01043524.

40. Lesieur HR, Klein R. Pathological gambling among high school students. Addict Behav. 1987; https://doi.org/10.1016/0306-4603(87)90019-0.

41. Eckstrom C. Adolescent gambling study. Alberta Alcohol and Drug Abuse Commission: Lethbridge; 1996

42. Wynne HJ, Smith GJ, Jacobs DF. Adolescent gambling and problem gambling in Alberta. Final report. Edmonton: Alberta drug and alcohol drug abuse commission; 1996. https://doi.org/10.5072/PRISM/9365

43. Fisher S. A prevalence study of gambling and problem gambling in British adolescents. Addict Res. 1999; https://doi.org/10.3109/ 16066359909004403. 
44. Ladouceur R, Boudreault N, Jacques C, Vitaro F. Pathological gambling and related problems among adolescents. J Child Adolesc Subst Abuse. 1999; https://doi.org/10.1300/J029v08n04_04.

45. Poulin C. Problem gambling among adolescent students in the Atlantic provinces of Canada. J Gambl Stud. 2000; https://doi.org/10.1023/A: 1009431417238

46. Adlaf EM, lalomiteanu A. Prevalence of problem gambling in adolescents: Findings from the 1999 Ontario Student Drug Use Survey. Can J Psychiatry. 2000. https://doi.org/10.1177/070674370004500809.

47. Westphal JR, Rush JA, Stevens L, Johnson LJ. Gambling behavior of Louisiana students in grades 6 through 12. Psych Serv. 2000. https://doi.org/ 10.1176/ps.51.1.96.

48. Hardoon KK, Derevensky JL. Social influences involved in children's gambling behavior. J Gambl Stud. 2001; https://doi.org/10.1023/A: 1012216305671.

49. Shaffer HJ, Hall MN, Vander Bilt JV. Estimating the prevalence of disordered gambling in the United States and Canada: a meta-analysis. Cambridge: Harvard Medical School Division of Addictions; 1997.

50. Ladouceur R. The prevalence of pathological gambling in Canada. J Gambl Stud. 1996; https://doi.org/10.1007/BF01539170.

51. Volberg RA. Gambling and problem gambling among adolescents in New York. New York Council on Problem Gambling 1998. https://doi.org/10. 5072/PRISM/9527. Accessed 10 Apr 2019.

52. Gupta R, Derevensky JL. Adolescent gambling behavior: a prevalence study and examination of the correlates associated with problem gambling. J Gambl Stud. 1998; https://doi.org/10.1023/A:1023068925328.

53. Sheela PS, Choo W, Goh LY, Phoay Lay Tan C. Gambling Risk Amongst Adolescents: Evidence from a School-Based Survey in the Malaysian Setting. J Gambl Stud. 2016; https://doi.org/10.1007/s10899-015-9577-3.

54. Di Nicola M, Ferri VR, Moccia L, Panaccione I, Strangio AM, Tedeschi D, Grandinetti P, Callea A, De-Giorgio F, Martinotti G, Janiri L. Gender differences and psychopathological features associated with addictive behaviors in adolescents. Front Psychiatry. 2017; https://doi.org/10.3389/fpsyt.2017.00256.

55. Livazović G, Bojčić K. Povezanost sociodemografskih obilježja, rizičnih stilova ponašanja i sklonosti kockanju adolescenata/the connection between sociodemographic features, risk behavioural styles and tendencies of adolescent gambling. Život i škola. 2017;63(1):137-50 Available at: https:// hrcak.srce.hr/193887. Accessed 1 Mar 2018.

56. Elton-Marshall T, Leatherdale ST, Turner NE. An examination of internet and land-based gambling among adolescents in three Canadian provinces: results from the youth gambling survey (YGS). BMC Public Health. 2016; https://doi.org/10.1186/s12889-016-2933-0.

57. Mutti-Packer S, Hodgins DC, El Guebaly N, Casey DM, Currie SR, Williams RJ, Smith GJ, Schopflocher DP. Problem Gambling Symptomatology and Alcohol Misuse Among Adolescents: A ParallelProcess Latent Growth Curve Model. Psychol Addict Behav. 2017; https://doi.org/10.1037/adb0000261.

58. Gonzalez-Roz A, Fernandez-Hermida J, Weidberg S, Martınez-Loredo V, Secades-Villa R. Prevalence of problem gambling among adolescents: a comparison across modes of access, gambling activities, and levels of severity. J Gambl Stud. 2017; https://doi.org/10.1007/s10899-016-9652-4.

59. Wilber, K. M., Potenza, M. Adolescent gambling: Research and clinical implications. Psychiatry. 2006; (Edgmont (Pa: Township). 3. 40-8., Available at: https://www.researchgate.net/publication/46579639_ Adolescent_gambling_Research_and_clinical_implications. Accessed 10 June 2019.

60. McBride J, Derevensky J. Gambling and Video Game Playing Among Youth. J Gambl Iss. 2016. https://doi.org/10.4309/jgi.2016.34.9.

61. Kristiansen SG, Jensen SM. Prevalence and correlates of problematic gambling among Danish adolescents. Int J Soc Welf. 2014; https://doi.org/ 10.1111/ijsw.12021.

62. Reith G. Research on the social impacts of gambling: Final report. Scottish Executive Social Research. 2006. available online at: https://www.gla.ac.uk/ media/media_34552_en.pdf. Accessed 7 Jun 2019.

63. Burge AN, Pietrzak RH, Petry NM. Pre/early adolescent onset of gambling and psychosocial problems in treatment-seeking pathological gamblers. J Gambl Stud. 2006. https://doi.org/10.1007/s10899-006-9015-7.

64. Lloyd J, Doll H, Hawton K, Dutton WH, Geddes JR, Goodwin GM, Rogers RD. How psychological symptoms relate to different motivations for gambling: an online study of internet gamblers. Biol Psychiatry. 2010. https://doi.org/ 10.1016/j.biopsych.2010.03.038
65. Yip SW, Desai RA, Steinberg MA, Rugle L, Cavallo DA, Krishnan-Sarin S, Potenza MN. Health/functioning characteristics, gambling behaviors, and gambling-related motivations in adolescents stratified by gambling problem severity: findings from a high school survey. Am J Addict. 2011; https://doi. org/10.1111/j.1521-0391.2011.00180.x.

66. Foster DW, Hoff RA, Pilver CE, Yau YHC, Steinberg MA, Wampler J, KrishnanSarin S, Potenza MN. Correlates of gambling on high-school grounds. Addict Behav. 2015. https://doi.org/10.1016/j.addbeh.2015.07.006.

67. Dodig D, Ricijaš N. Obilježja kockanja zagrebačkih adolescenata. Ljetopis socijalnog rada. 2011;18(1):103-25.

68. Canale N, Vieno A, ter Bogt T, Pastore M, Siciliano V, Molinaro S. Adolescent gambling-oriented attitudes mediate the relationship between perceived parental knowledge and adolescent gambling: implications for prevention. Prev Sci. 2016. https://doi.org/10.1007/s11121-016-0683-y.

69. Jacobs DF, Marston AR, Singer RD, Widaman K, Little T, Veizades J. Children of problem gamblers. J Gambl Stud. 1989. https://doi.org/10. 1007/BF01672427.

70. Griffiths M, Wood RTA. Risk factors in adolescence: the case of gambling, videogame playing and the internet. J Gambl Stud. 2000; https://doi.org/10. 1023/A:1009433014881.

71. Griffiths M. Toward a risk factor model of fruit machine addiction: a brief note. J Gambling Stud. 1995. https://doi.org/10.1007/BF02104797.

72. Rossen FV, Clark T, Denny SJ, Fleming TM, Peiris-John R, Robinson E, Lucassen MFG. Unhealthy gambling amongst New Zealand secondary school students: an exploration of risk and protective factors. Int J Ment Health Addiction. 2016; https://doi.org/10.1007/s11469-015-9562-1.

73. Castrén S, Grainger M, Lahti T, Alho H, Salonen AH. At-risk and problem gambling among adolescents: a convenience sample of first-year junior high school students in Finland. Subst Abuse Treat Prev Policy. 2015. https://doi.org/10.1186/s13011-015-0003-8.

74. Vitaro F, Brendgen M, Ladouceur R, Tremblay RE. Gambling, delinquency, and drug use during adolescence: mutual influences and common risk factors. J Gambl Stud. 2001. https://doi.org/10.1023/A: 1012201221601

75. Allami Y, Vitaro F, Brendgen M, Carbonneau R, Tremblay RE. Identifying atrisk profiles and protective factors for problem gambling: a longitudinal study across adolescence and early adulthood. Psychol Addict Behav. 2018; https://doi.org/10.1037/adb0000356.

76. Jackson AC, Dowling N, Thomas SA, Bond L, Patton G. Adolescent gambling behaviour and attitudes: a prevalence study and correlates in an Australian population. Int J Ment Health Addiction. 2008; https://doi.org/10.1007/ s11469-008-9149-1.

77. Hardoon K, Derevensky JL, Gupta R. Empirical measures vs. perceived gambling severity among youth: why adolescent gamblers fail to seek treatment. Addict Behav. 2003;28:933-46.

78. Volberg R, Gupta R, Griffiths MD, Olason DT, Delfabbro P. An international perspective on youth gambling prevalence studies. Int J Adolesc Med Health. 2010; https://doi.org/10.1515/IJAMH.2010.22.1.3

79. Smith N. Psychosocial and Neurobiological Factors in the Development of Gambling Disorders. In: Reilly C, Kugler A, Smith N, Mayes NP, editors. Increasing the odds: A series dedicated to understanding gambling disorders, volume 7 what clinicians need to know about gambling disorders. Washington: National center for responsible gaming. p. 5-13. http://www.ncrg.org/sites/default/files/uploads/docs/ monographs/ncrgmonograph7final.pdf.

80. Bergevin, T., Gupta, R., Derevensky, J., Kaufman F. Adolescent gambling: Understanding the role of stress and coping. J Gambl Stud 2006; Epub, 2006. Available at: http://springerlink.metapress.com/(r4c1ic45k5pyvt45 yd00x0qg)/app/home/issue.asp?referrer=parent\&backto=journal,1,81; linkingpublicationresults, 1:105582,1.

81. Gupta R, Derevensky JL. An empirical examination of Jacobs' general theory of addictions: do adolescent gamblers fit the theory? J Gambl Stud. 1998; https://doi.org/10.1023/A:1023046509031.

82. Schreiber L, Odlaug BL, Kim SW, Grant JE. Characteristics of pathological gamblers with a problem gambling parent. Am J Addict. 2009; https://doi. org/10.3109/10550490903206007.

83. Ferland F, Ladouceur R, Vitaro F. Prevention of problem gambling: modifying misconceptions and increasing knowledge. J Gambl Stud. 2002;18(1):19-29.

84. Gaboury A, Ladouceur R. Evaluation of a prevention program for pathological gambling among adolescents. J Prim Prev. 1993;14:21-8. 
85. Smith GT, McCarthy DM, Goldman MS. Self-reported drinking and alcohol-related problems among early adolescents: dimensionality and validity over 24 months. J Stud Alcohol. 1995; https://doi.org/10.15288/ jsa.1995.56.383.

86. Stanton WR, McClelland M, Elwood C, Ferry D, Silva PA. Prevalence, reliability and bias of adolescents' reports of smoking and quitting. Addiction. 1996; https://doi.org/10.1046/j.1360-0443.1996.9111170511.x.

\section{Publisher's Note}

Springer Nature remains neutral with regard to jurisdictional claims in published maps and institutional affiliations.

Ready to submit your research? Choose BMC and benefit from:

- fast, convenient online submission

- thorough peer review by experienced researchers in your field

- rapid publication on acceptance

- support for research data, including large and complex data types

- gold Open Access which fosters wider collaboration and increased citations

- maximum visibility for your research: over $100 \mathrm{M}$ website views per year

At $\mathrm{BMC}$, research is always in progress.

Learn more biomedcentral.com/submissions 